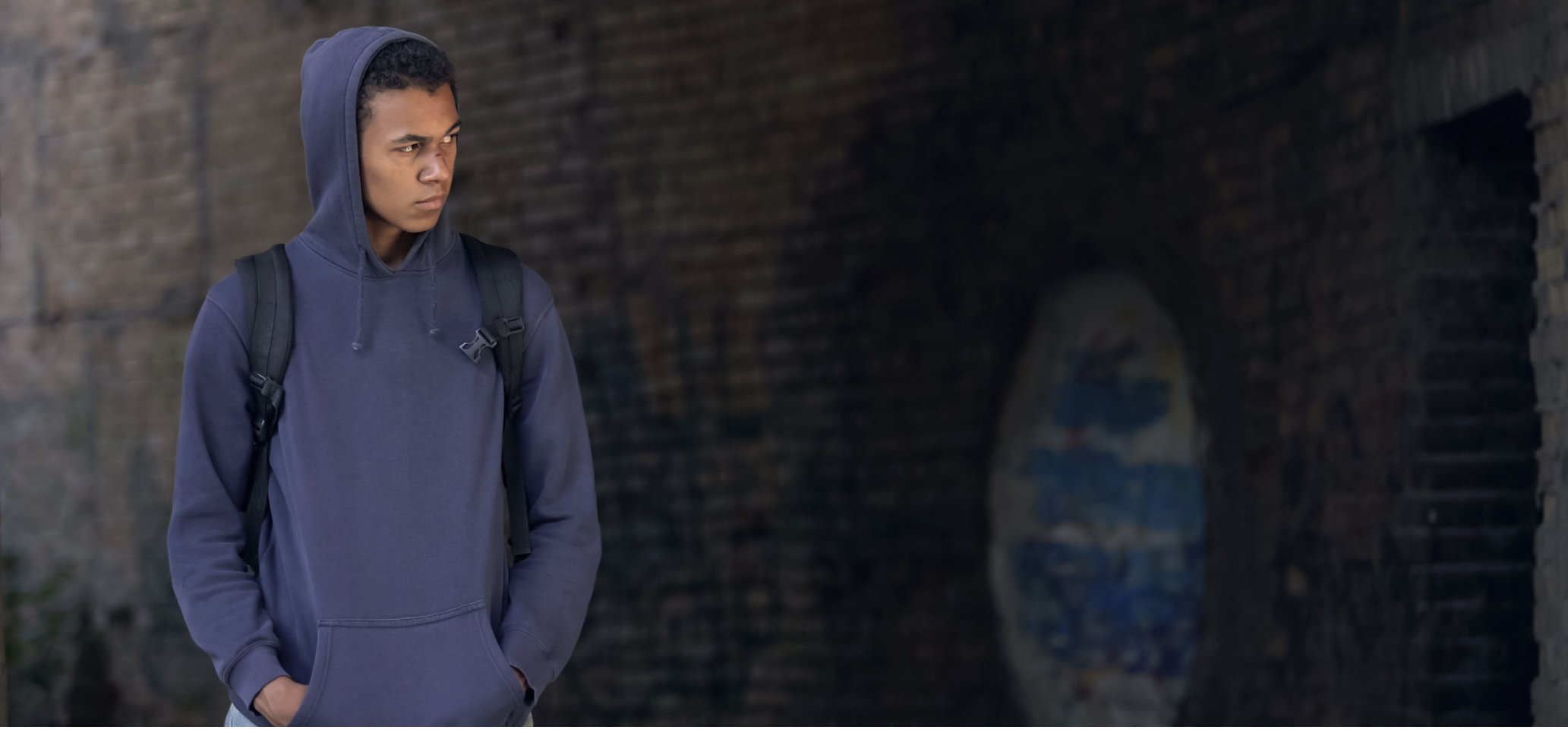

\title{
Early caregiving experiences shape adolescent attachment profiles
}

By Jessica K. Edwards

Secure attachment in adolescents seems to be associated with robust mental health and social skills ${ }^{1,2}$. How the quality of early caregiving impacts on attachment security in adolescence, however, is less clear. In 2019, Thomas O'Connor and colleagues addressed this question through two longitudinal studies on the relative contribution of early and current caregiving quality to attachment security in adolescence. Here, they measured the quality of parent-child relationships at age 3-7 years and parent-adolescent relationship quality at $\sim 12$ years in a clinic-referred and an at-risk community sample of adolescents. They found that observational ratings of both early childhood and current caregiving quality were significantly associated with adolescent attachment security. Only early caregiver quality, however, could independently predict adolescent attachment patterns. This longitudinal prediction was stronger in the at-risk community sample than in the clinic sample; the reason for this difference, however, requires further investigation. Taken together, these findings imply a strong role for the influence of caregiving in early childhood, not in adolescence, on the formation of adolescent attachment representations. The researchers propose that early caregiving quality might be targeted to help shape adolescents' relationship representations and promote mental health well-being and adjustment.

\section{Referring to:}

O'Connor, T.G., Woolgar, M., Humayun, S., Briskman, J.A. \& Scott, S. (2019), Early caregiving predicts attachment representations in adolescence: findings from two longitudinal studies. J. Child Psychol. Psychiatr. 60: 944-952. doi: 10.111/jcpp.12936.

\section{References:}

${ }^{1}$ Cavendish, W. et al. (2012), Parent attachment, school commitment, and problem behaviour trajectories of diverse adolescents. J. Adolesc. 35: 1629-1639. doi: 10.1016/j.adolescence.2012.08.001.

${ }^{2}$ Allen, J.P. et al. (2007), The relation of attachment security to adolescents' paternal and peer relationships, depression, and externalizing behavior. Child Dev. 78: 1222-1239. doi: 10.1111/j.1467-8624.2007.01062.x 